\title{
Positrons and Positronium Atoms in a Condensed Phase Close to Its Melting Point
}

\author{
S.V. Stepanov ${ }^{a, b, *}$, D.S. ZvezhinskiY ${ }^{a}$, V.M. ByAKOV ${ }^{a, c}$, G. Duplâtre $^{d}$ \\ AND P.S. STEPANOV ${ }^{a, b}$
}

${ }^{a}$ Institute for Theoretical and Experimental Physics, B. Cheremushkinskaya 25, 117218, Moscow, Russia

${ }^{b}$ National Research Nuclear University "MEPhI", Kashirskoye shosse 31, Moscow, 115409, Russia

${ }^{c}$ D. Mendeleyev University of Chemical Technology of Russia, Miusskaya sq. 9, 125047, Moscow, Russia

${ }^{d}$ Institut Pluridisciplinaire Hubert Curien, CNRS/IN2P3, BP 2867037 Strasbourg, Cedex 2, France

\begin{abstract}
Investigation of the local heating effect at the terminal part of a fast positron track sheds light on the accumulation of defects in a frozen medium close to its melting point. Estimations of the formation energies of the structural defects which may trap the Ps atom as well as a contribution of these defects to the enthalpy of fusion are obtained.
\end{abstract}

DOI: $10.12693 /$ APhysPolA.125.691

PACS: 71.60.+z, 34.80.Lx, 78.70.Bj, 82.30.Gg

\section{Introduction}

It is well known that positrons $\left(\mathrm{e}^{+}\right)$as well as positronium atoms $(\mathrm{Ps})$ are convenient nanoscale probes of the local nanoscale structure in a condensed phase and the radiolytic processes occurring therein [1]. The sensitivity to the presence of structural defects opens a possibility to study the nature of phase transitions and, particularly, the accumulation of defects at a stage of premelting, one of the fundamental and puzzling phenomena in physics.

\section{Track structure of a fast positron}

Positrons, produced in nuclear $\beta^{+}$-decay, have initial energies of about several hundreds of $\mathrm{keV}$. Once injected into a medium they lose energy via molecular ionization. Within 10 ps the positron energy drops down to the ionization threshold, Fig. 1.

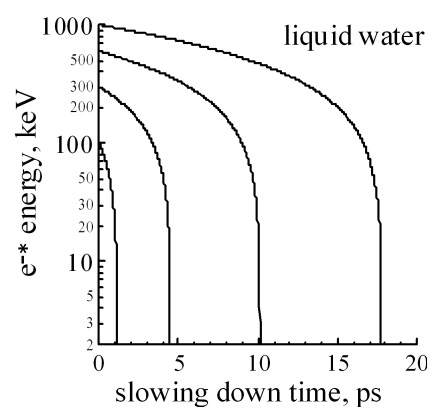

Fig. 1. Typical times of ionization slowing down scale of $\mathrm{e}^{+} / \mathrm{e}^{-}$having initial kinetic energy $0.1,0.3,0.6$ and $1 \mathrm{MeV}$ in liquid water (calculated on the basis of slowing down data http://physics.nist.gov/ PhysRefData/Star/Text/ESTAR-u.html).

*corresponding author; e-mail: stepanov@itep.ru
When the $\mathrm{e}^{+}$energy becomes less than the blob formation energy, $W_{\mathrm{bl}}(<1 \mathrm{keV}$ or so), the positron is about to create a terminal blob [2]. The diffusion motion of $\mathrm{e}^{+}$in the blob becomes more pronounced: the direction of its momentum changes frequently due to elastic scattering and ionization of surrounding molecules. All intrablob ionizations are confined within a sphere of radius $a_{\mathrm{bl}}$. The terminal positron blob contains a few tens of ion-electron pairs $\left(n_{0} \sim W_{\mathrm{bl}} / W_{\mathrm{iep}} \approx 30\right)$ because the average energy $W_{\text {iep }}$ required to produce one ion-electron pair is $16-22 \mathrm{eV}$. The formation of the terminal blob lasts $\approx 0.01$ ps. Further approach towards thermal equilibrium (from $\approx 10 \mathrm{eV}$ down to $k_{\mathrm{B}} T$ ) proceeds primarily via excitations of intra- and intermolecular vibrations and usually takes a few tens of femtoseconds. The ultimate stage of transformation of the projectile positron as well as of secondary track electrons is solvation (in water hydration). The electron hydration time is about $0.3 \mathrm{ps}$.

\section{Thermal spikes in the $\mathrm{e}^{+}$terminal blob}

For the first time we have pointed out the effect of local heating of the terminal part of the positron track, on the basis of positron annihilation lifetime (PAL) experiments in octanol [3]. Close to the melting point the data show a so to say "preliminary" formation of the so-called Ps bubble state (it is a common standpoint that in a liquid state Ps atom forms a nanobubble in which it resides). While the major fraction of the medium is solid, the Ps bubble may be formed within a premelted region close to the origin of the $\mathrm{e}^{+}$blob. A rapid increase in the long-lived (ortho-Ps) lifetime, $\tau_{3}$ (as well as a decrease in intensity $I_{3}$ of this lifetime component) from its solid phase value to the value corresponding to the liquid state occurs at a temperature a little bit lower than the melting point of octanol, Fig. 2. However no attention was paid to this observation.

When the temperature of the frozen medium is only slightly below its melting point, almost all the energy re- 


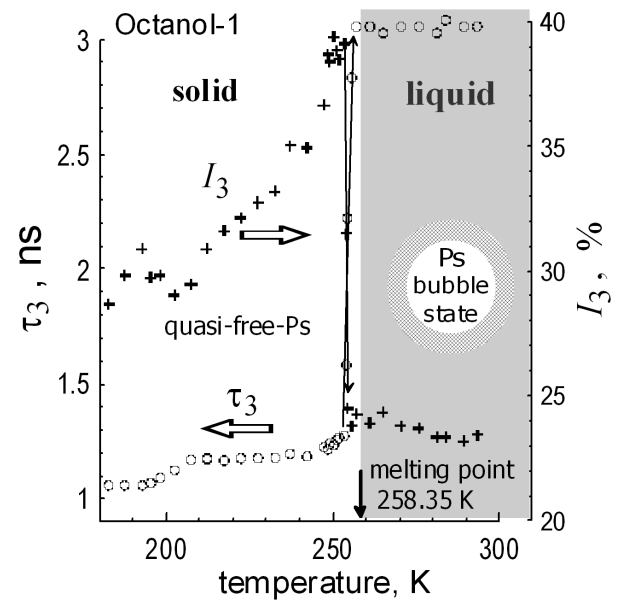

Fig. 2. Temperature dependences of the ortho-Ps lifetime and of the intensity of the corresponding lifetime component in octanol [3].

leased in the $\mathrm{e}^{+}$blob is spent on the enthalpy of fusion and due to the subsequent local heating of the liquid phase is formed. However, the size of the molten volume is comparable to the initial size of the $\mathrm{e}^{+}$blob. In the molten region the Ps atom can form a bubble state, in spite of the bulk of the medium remaining solid at a temperature below the melting point.

Figure 3 illustrates the kinetics of local heating in liquid water at a temperature slightly higher than $0{ }^{\circ} \mathrm{C}$, and in ice at $-4{ }^{\circ} \mathrm{C}$ (it was assumed that the slowing down positron deposits $1 \mathrm{keV}$ of its kinetic energy, which converts into heat). It is clearly seen from the bottom pair of graphs in Fig. 3 that (1) the positron heats ice up to $0{ }^{\circ} \mathrm{C},(2)$ the temperature of the blob reaches a plateau at $0^{\circ} \mathrm{C}$ (at this stage, the energy deposited is spent on latent heat of fusion), (3) the central part of the blob becomes molten. After $\approx 20$ ps the molten region becomes frozen again and the accumulated latent heat of fusion is slowly transferred to the bulk of the medium. Since the temperature gradient is small in the region between the heated region and the bulk of the medium, thermal spikes in ice live longer than in liquid water.

The phenomenological description of the local heating effect as a function of time is given by solving the heat transfer equation

$$
\begin{aligned}
& c_{p} \rho \frac{\partial T(r, t)}{\partial t}=\operatorname{div}(\lambda \nabla T)+q_{+}(r, t), \\
& T(r, t=0)=T_{\mathrm{b}},
\end{aligned}
$$

where $T(r, t)$ is the local temperature, $T_{\mathrm{b}}$ is the temperature of the medium far from the blob and $q_{+}$describes heat generation due to ionization by the slowing down positron. $q_{+}(r, t)$ is proportional to a product of two Gaussians: over $t$, centered at $t=1 \mathrm{ps}$ with FWHM $=0.7 \mathrm{ps}$, and over $r$, centered at $r=0$ with $\mathrm{FWHM}=100 \AA$. The meaning of the other symbols is as usual. The heat capacity $c_{p}$ is temperature dependent. Below the melting point it equals to that in the
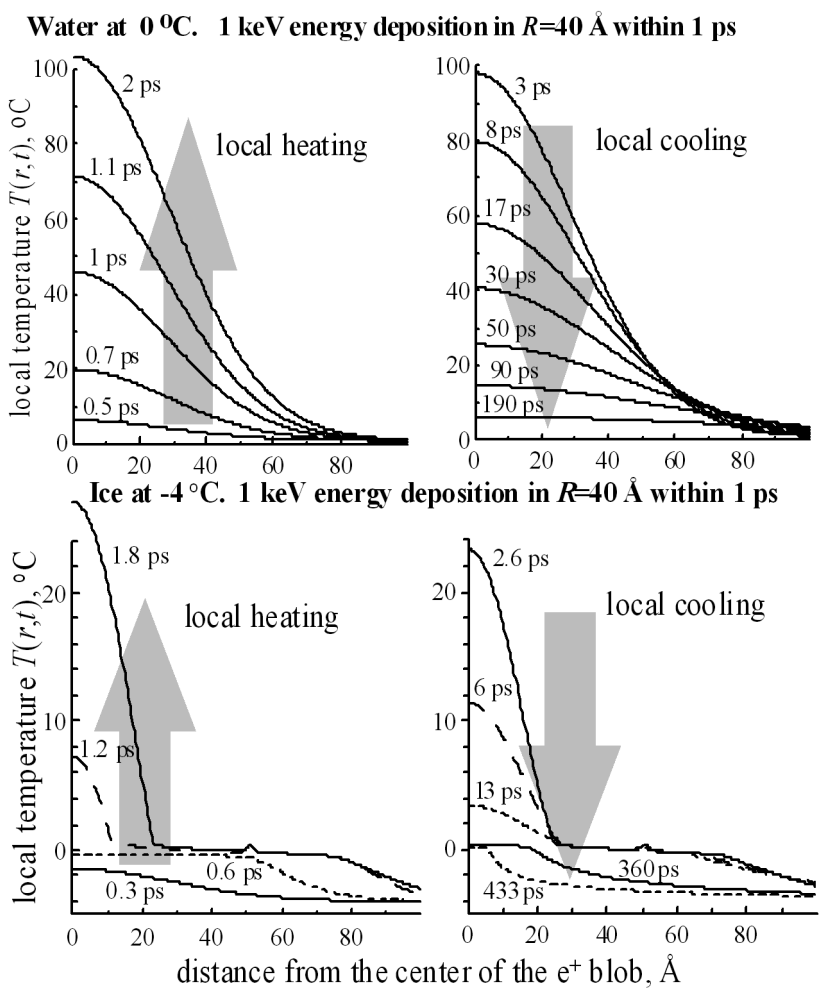

Fig. 3. Thermal spikes in the $\mathrm{e}^{+}$blob in water (at $T_{\mathrm{b}}=0{ }^{\circ} \mathrm{C}$ ) and in ice $\left(\right.$ at $T_{\mathrm{b}}=-4^{\circ} \mathrm{C}$ ). Solid lines represent solutions of the heat transfer Eq. (1). The energy $(1 \mathrm{keV})$ arising from the $\mathrm{e}^{+}$slowing down is released within 1 ps (Gaussian distribution function of time).

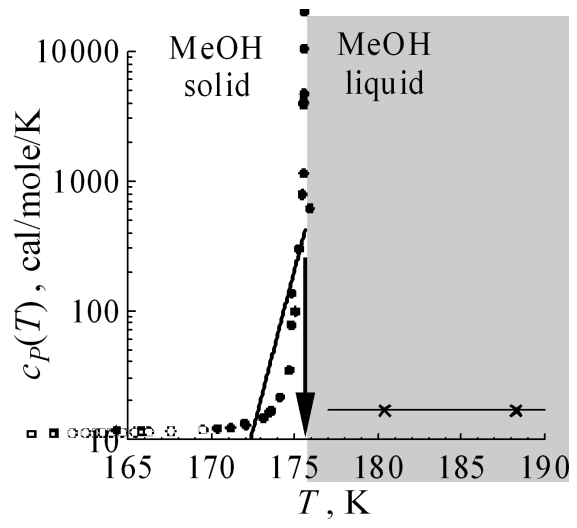

Fig. 4. Heat capacity of methanol vs. temperature [5].

solid phase, $c_{p}^{\mathrm{S}}\left(T_{\mathrm{b}}\right)$, above $T_{\mathrm{m}}$ it equals $c_{p}^{\mathrm{L}}\left(T_{\mathrm{b}}\right)$ and in the vicinity of $T_{\mathrm{m}}$ the heat capacity contains an additional quasi-singular term, which takes into account the absorption of the latent heat and is approximated by a narrow Gaussian with the width $\Delta T \approx 1^{\circ} \mathrm{C}[4]$ :

$$
H_{\mathrm{m}} \exp \left(-\frac{\left(T-T_{\mathrm{m}}\right)^{2}}{2 \Delta T^{2}}\right) / \sqrt{2 \pi \Delta T^{2}} .
$$

Integrating this term over temperature gives the specific enthalpy of fusion $H_{\mathrm{m}}$. As an example, Fig. 4 shows the 

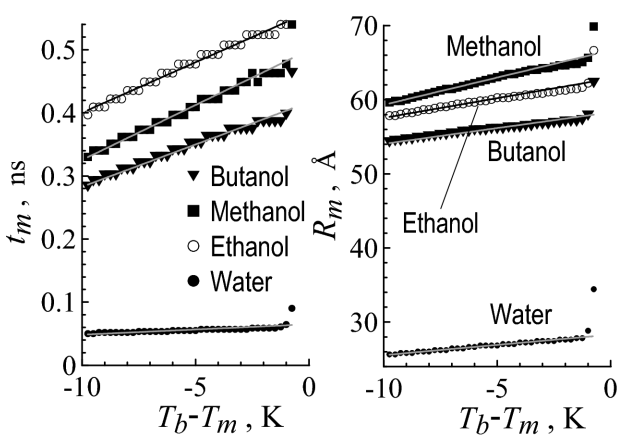

Fig. 5. The lifetimes $t_{\mathrm{m}}$ of the molten regions (left) and their maximal radii $R_{\mathrm{m}}$ (right) in methanol, ethanol, butanol and water. All quantities are displayed as a function of the difference between the bulk temperature $\left(T_{\mathrm{b}}\right)$ and the melting temperature $\left(T_{\mathrm{m}}\right)$.

temperature dependence of the specific heat capacity of methanol close to its melting temperature [5].

For thermal conductivity $\lambda$ and density $\rho$ we have used smooth interpolations linking their values in the solid and liquid phases $\lambda^{\mathrm{S}}, \lambda^{\mathrm{L}}$, and $\rho^{\mathrm{S}}, \rho^{\mathrm{L}}$, respectively.

Solving numerically Eq. (1), we have obtained temperature profiles as shown in Fig. 3 and also the maximal radii $\left(R_{\mathrm{m}}\right)$ of the molten regions in different media and the times $\left(t_{\mathrm{m}}\right)$ when these molten areas reach their maxi- mal volumes, Fig. 5. In the calculations we used the data from the Table (see Refs. in [2]).

In Ref. [6], we have tried to visualize the local melting effect which takes place in the terminal part of the positron track ( $\mathrm{e}^{+}$blob) by studying the temperature dependences of the ortho-Ps lifetime in alcohols, Fig. 6 .

It is seen from Fig. 6 that similarly to the case of octanol (Fig. 2) the formation of the Ps bubble states takes place a few degrees below the melting temperatures of the bulk.

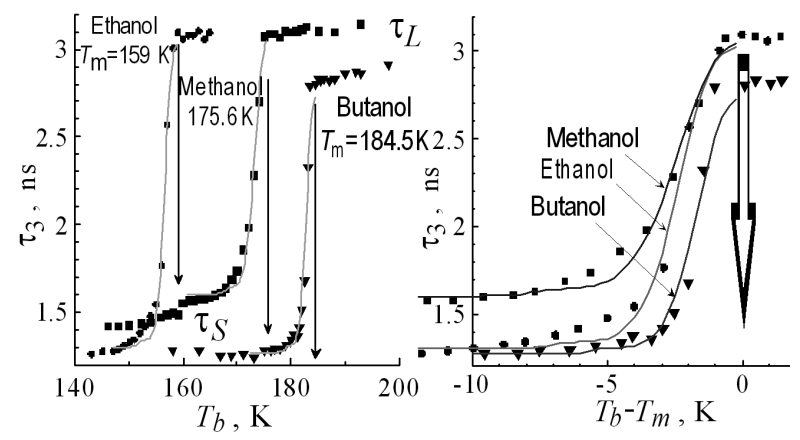

Fig. 6. Temperature dependences of the long-lived (ortho-Ps) component of the PAL spectra in alcohols near their melting temperatures (data from [6]).

Numerical values of the parameters.

TABLE

\begin{tabular}{|c|c|c|c|c|c|c|}
\hline Substance & $T_{\mathrm{m}}[\mathrm{K}]$ & $\begin{array}{c}\lambda^{\mathrm{S}} ; \lambda^{\mathrm{L}} \\
{[\mathrm{W} /(\mathrm{m} \mathrm{K})]}\end{array}$ & $\begin{array}{c}H_{\mathrm{m}} \\
{[\mathrm{J} / \mathrm{g}]}\end{array}$ & $c_{p}^{\mathrm{S}} ; c_{p}^{\mathrm{L}}[\mathrm{J} /(\mathrm{g} \mathrm{K})]$ & $\begin{array}{c}\rho^{\mathrm{S}} ; \rho^{\mathrm{L}} \\
{\left[\mathrm{g} / \mathrm{cm}^{3}\right]}\end{array}$ & $M[g]$ \\
\hline methanol & 175.6 & $0.32 ; 0.21$ & 99.2 & $\begin{array}{l}333.75-3.743 T_{\mathrm{b}}+0.0122 T_{\mathrm{b}}^{2} \\
100.9-0.322 T_{\mathrm{b}}+0.000866 T_{\mathrm{b}}^{2}\end{array}$ & $0.98 ; 0.79$ & 32.04 \\
\hline ethanol & 159 & $0.27 ; 0.17$ & 209 & $\begin{array}{c}-8.12+1.166 T_{\mathrm{b}}-0.0092 T_{\mathrm{b}}^{2}+0.0000308 T_{\mathrm{b}}^{3} \\
88.45+0.047 T_{\mathrm{b}}-0.00083 T_{\mathrm{b}}^{2}+0.000003 T_{\mathrm{b}}^{3}\end{array}$ & $1.06 ; 0.79$ & 46.07 \\
\hline butanol & 184.5 & $0.47 ; 0.16$ & 109 & $\begin{array}{c}-88.07+3.285 T_{\mathrm{b}}-0.027 T_{\mathrm{b}}^{2}+0.00011 T_{\mathrm{b}}^{3} \\
63.05+0.983 T_{\mathrm{b}}-0.00517 T_{\mathrm{b}}^{2}+10^{-5} T_{\mathrm{b}}^{3}\end{array}$ & $1.05 ; 0.81$ & 74.12 \\
\hline water & 273.15 & $2.38 ; 0.56$ & 334 & $2.06 ; 4.18$ & $0.92 ; 1.00$ & 18 \\
\hline
\end{tabular}

\section{Quantitative interpretation}

For quantitative interpretation of the experimental data, we have assumed that:

1) a quasi-free Ps atom ( $q$ f-Ps) is formed as a result of combination of a thermalized positron with one of the intratrack electrons (the diffusion-recombination mechanism):

$$
\mathrm{e}^{+}+\mathrm{e}^{-} \rightarrow \text { qf-Ps. }
$$

The formation probability of qf-Ps is $P_{\mathrm{qf}-\mathrm{Ps}_{\mathrm{s}}}$. The distribution of qf-Ps in space and time may be approximated as:

$$
\begin{aligned}
& c_{\mathrm{qf}-\mathrm{Ps}}(r, t)=P_{\mathrm{qf}-\mathrm{Ps}} \exp \left(-\lambda_{\mathrm{v}} t\right) G(r, t), \\
& G(r, t)=\frac{\exp \left(-r^{2} / 4 D_{\mathrm{Ps}} t\right)}{\pi^{3 / 2}\left(a^{2}+4 D_{\mathrm{Ps}} t\right)^{3 / 2}},
\end{aligned}
$$

where $a$ is the initial size of the $\mathrm{e}^{+}$blob, $D_{\mathrm{Ps}}=$ $v_{\mathrm{Ps}} \lambda_{\mathrm{Ps}} / 6 \approx \hbar /\left(6 m_{\mathrm{Ps}}\right) \approx 0.1 \mathrm{~cm}^{2} / \mathrm{s}$ is the diffusion coefficient of qf-Ps and $\lambda_{\mathrm{v}}\left(T_{\mathrm{b}}\right)$ is the qf-Ps localization (or trapping) rate constant in structural traps in a solid phase (for the sake of simplicity, we shall call these traps as "vacancies"; their concentration increases very rapidly when approaching the melting point). So we assume that qf-Ps transforms into positronium, localized in a vacancy $(\mathrm{v}-\mathrm{Ps})$ :

$$
\text { qf-Ps } \stackrel{\lambda_{\mathrm{v}}}{\longrightarrow} \mathrm{v}-\mathrm{Ps} \text {. }
$$

The distribution of $\mathrm{v}$-Ps in space and time may be obtained by integrating Eq. (3) over time

$$
c_{\mathrm{v}-\mathrm{PS}}(r, t)=\lambda_{\mathrm{v}}\left(T_{\mathrm{b}}\right) \int_{0}^{t} c_{\mathrm{qf}-\mathrm{Ps}}\left(r, t^{\prime}\right) \mathrm{d} t^{\prime} .
$$

For the trapping rate $\lambda_{\mathrm{v}}$ one may use the following stan- 
dard expression typical for diffusion-controlled reactions:

$$
\lambda_{\mathrm{v}}\left(T_{\mathrm{b}}\right)=4 \pi D_{\mathrm{Ps}} R_{\mathrm{Ps}} c_{\mathrm{v}}\left(T_{\mathrm{b}}\right), \quad R_{\mathrm{Ps}} \approx 10 \AA .
$$

Here, the strongest temperature-dependent quantity is the concentration of vacancies $c_{\mathrm{v}}\left(T_{\mathrm{b}}\right)$. We shall assume that it obeys the Arrhenius law

$$
c_{\mathrm{v}}\left(T_{\mathrm{b}}\right) \sim \exp \left(-E_{\mathrm{v}} / k_{\mathrm{B}} T_{\mathrm{b}}\right) .
$$

It ensues that $\lambda_{\mathrm{v}}\left(T_{\mathrm{b}}\right)$ also obeys the Arrhenius distribution;

2) if a qf-Ps is localized near the center of the $\mathrm{e}^{+}$blob and thus a v-Ps state is created at $r<R_{\mathrm{m}}$, then by the time $t_{\mathrm{m}}$ this area will be molten and this $\mathrm{v}-\mathrm{Ps}$ forms a bubble state with lifetime $\tau_{\mathrm{L}}=2.8-3 \mathrm{~ns}$, which is typical of the liquid phase. If a qf-Ps is localized outside $R_{\mathrm{m}}$, such a v-Ps will be living in a solid phase and finally annihilate within the characteristic time $\tau_{\mathrm{S}}=1.3-1.6 \mathrm{~ns}$;

3 ) the most important $T$-dependent quantity is the qf-Ps trapping rate $\lambda_{\mathrm{v}}\left(T_{\mathrm{b}}\right)$. This is fortunate for PALS, because it gives us a possibility to study the accumulation of defects near to a phase transition. After cooling, the molten region of the blob becomes frozen and the Ps bubble state turns out to be frozen as well, keeping the same lifetime as in the liquid phase. The fraction of Ps atoms which are transformed into the bubble state (b-Ps) is

$$
P_{\mathrm{b}-\mathrm{Ps}}\left(T_{\mathrm{b}}\right)=\int_{r<R_{\mathrm{m}}}\left[c_{\mathrm{qf}-\mathrm{Ps}}\left(r, t_{\mathrm{m}}\right)+c_{\mathrm{v}-\mathrm{Ps}}\left(r, t_{\mathrm{m}}\right)\right] \mathrm{d}^{3} r .
$$

The rest of the Ps atoms, outside of the molten region will end up captured in vacancies. Therefore, the experimentally observable temperature dependence of the lifetime $\tau_{3}$ of the long-lived component of the PAL spectrum (the lifetime of the Ps bubble state) can be calculated as follows:

$$
\frac{1}{\tau_{3}\left(T_{\mathrm{b}}\right)}=\frac{1}{\tau_{\mathrm{S}}}+\left(\frac{1}{\tau_{\mathrm{L}}}-\frac{1}{\tau_{\mathrm{S}}}\right) \frac{P_{\mathrm{b}-\mathrm{Ps}}\left(T_{\mathrm{b}}\right)}{P_{\mathrm{qf}-\mathrm{Ps}}} .
$$

The experimental data in Fig. 6 were treated with the help of the above expression. The result is shown in solid lines. We could derive the formation energies $E_{\mathrm{v}}$ of the vacancy-type defects ( $3 \mathrm{eV}$ for methanol and ethanol and $5 \mathrm{eV}$ for butanol) together with $V$ in the corresponding pre-exponential factors $\exp (V)$ in the trapping rate $\lambda_{\mathrm{v}}\left[\mathrm{ns}^{-1}\right]=\exp \left(V-E_{\mathrm{v}} / k_{\mathrm{B}} T_{\mathrm{b}}\right):$

methanol: $E_{\mathrm{v}}=3 \mathrm{eV}, V=209$,

ethanol: $E_{\mathrm{v}}=3 \mathrm{eV}, V=230$,

butanol: $E_{\mathrm{v}}=5 \mathrm{eV}, V=325$.

Then it is interesting to answer the question: to what extent "our" defects contribute to the enthalpy of fusion and to the heat capacity? Let us multiply the energy $E_{\mathrm{v}}$ of the defect formation by the defect concentration at the melting temperature:

$$
\Delta H_{\mathrm{m}}=E_{\mathrm{v}} c_{\mathrm{v}}\left(T_{\mathrm{m}}\right)=E_{\mathrm{v}} \frac{\lambda_{\mathrm{v}}\left(T_{\mathrm{m}}\right)}{4 \pi D_{\mathrm{Ps}} R_{\mathrm{Ps}} \rho}[\mathrm{J} / \mathrm{g}] .
$$

By quantifying this expression in the case of methanol, one obtains $\Delta H_{\mathrm{m}} \approx 25 \mathrm{~J} / \mathrm{g}$, while the experimental value for the enthalpy of fusion is $99 \mathrm{~J} / \mathrm{g}$. Thus the structural vacancy-type defects (considered here) contribute to about one fourth of the experimental value of $H_{\mathrm{m}}$.
The contribution of these defects to the heat capacity may be estimated as follows:

$$
c_{\mathrm{P}}\left(T_{\mathrm{b}}\right)=E_{\mathrm{v}} \frac{\mathrm{d} c_{\mathrm{v}}\left(T_{\mathrm{b}}\right)}{\mathrm{d} T_{\mathrm{b}}} .
$$

This is illustrated in Fig. 4 by the solid straight line. Obviously, one can achieve a better agreement with the calorimetric data, taking into account the generation of other types of defects (not just "vacancies", but their associations and so on). This process is greatly accelerated when approaching the melting point. The equilibrium concentrations of these additional defects may be estimated on the basis of relationships similar to Eq. (6); their formation requires some additional energy and, therefore, the solid line in Fig. 4 will be bent closer to the experimental data.

Thus, one may say that the prevalent statement that absorption of the latent heat of melting supposedly takes place at constant temperature $\left(=T_{\mathrm{m}}\right)$ is not correct. In fact, we are dealing with very fast (avalanche-like) but equilibrium accumulation of various types of defects in the solid phase (in accordance with the Arrhenius law). When the bulk temperature approaches the melting point, the concentrations of defects grow, the internal local stress in the solid phase increases, reaches some critical level and the solid phase turns to liquid. All these processes (generation of defects and absorption of the latent heat) occur in a narrow (few degrees) temperature interval.

\section{Conclusions}

1) It is shown that the local heating effect is manifested in a specific behavior of the lifetime (and intensity) of the long-lived component of PAL spectra at temperatures close to the melting point. The lifetime reaches its liquid phase value when the bulk temperature is $2-3$ degrees below the melting point. Presumably this occurs due to local melting of the central part of the $\mathrm{e}^{+}$blob, where Ps atom may form the bubble state.

2) However, the experiments [6] cannot be considered as final, because it is necessary to proceed with a more accurate treatment of the raw PAL spectra based on the model we have developed (implying 4-exponential deconvolution of the spectra, not just 3-exponential as was assumed in [6]). Moreover, the intensities of the PAL time-components must also be included into the fitting procedure;

3) PAL data should be combined with calorimetric measurements (DSC), conducted on the same samples;

4) This work sheds light on the physics of melting, namely on premelting, when the effective generation of structural defects takes place in a solid phase. This process actually occurs in a narrow, but finite temperature range. Vibrations of molecules also contribute to the enthalpy of fusion and to the heat capacity, but it was not discussed here;

5) Of course, one cannot exclude the existence of a small scatter of the melting temperature itself because 
of some inhomogeneity of the frozen samples (polycrystallinity, grain boundaries).

\section{Acknowledgments}

This work was supported by the Russian Foundation for Basic Research (grant 11-03-01066).

\section{References}

[1] Positron and Positronium Chemistry, Eds. Y.C. Jean, P.E. Mallon, D.M. Schrader, World Sci., Singapore 2003.

[2] S.V. Stepanov, V.M. Byakov, D.S. Zvezhinskiy, G. Duplâtre, R.R. Nurmukhametov, P.S. Stepanov, Adv. Phys. Chem. 2012, 431962 (2012).
[3] F. Bockstahl, I. Billard, G. Duplâtre, A. Bonnenfant, Chem. Phys. 236, 393 (1998).

[4] M. Muhieddine, E. Canot, R. March, Int. J. Fin. 6, 1 (2009).

[5] H.G. Carlson, F. Edgar, Westrum, J. Chem. Phys. 54, 1464 (1971).

[6] D.S. Zvezhinskiy, S.V. Stepanov, V.M. Byakov, B. Zgardzińska, Mater. Sci. Forum 733, 15 (2013). 